\title{
Killed whole-genome reduced-bacteria surface-expressed coronavirus fusion peptide vaccines protect against disease in a porcine model
}

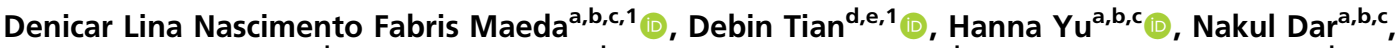

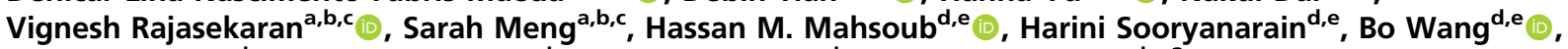

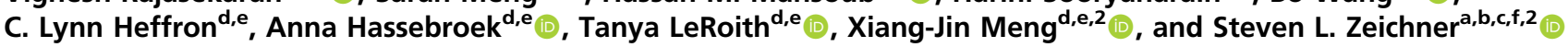

aDepartment of Pediatrics, University of Virginia, Charlottesville, VA 22908-0386; bendleton Pediatric Infectious Disease Laboratory, University of Virginia, Charlottesville, VA 22908-0386; ' ${ }^{C}$ Child Health Research Center, University of Virginia, Charlottesville, VA 22908-0386; ${ }^{\mathrm{d} D e p a r t m e n t}$ of Biomedical Sciences

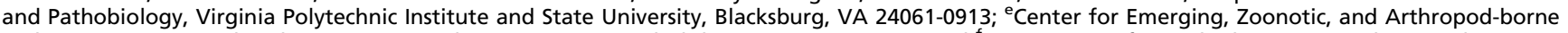
Pathogens, Virginia Polytechnic Institute and State University, Blacksburg, VA 24061-0913; and 'Department of Microbiology, Immunology, and Cancer Biology, University of Virginia, Charlottesville, VA 22908-0386

Contributed by Xiang-Jin Meng, February 14, 2021 (sent for review December 13, 2020; reviewed by Diego Diel and Qiuhong Wang)

As the coronavirus disease 2019 (COVID-19) pandemic rages on, it is important to explore new evolution-resistant vaccine antigens and new vaccine platforms that can produce readily scalable, inexpensive vaccines with easier storage and transport. We report here a synthetic biology-based vaccine platform that employs an expression vector with an inducible gram-negative autotransporter to express vaccine antigens on the surface of genome-reduced bacteria to enhance interaction of vaccine antigen with the immune system. As a proof-of-principle, we utilized genome-reduced Escherichia coli to express SARS-CoV-2 and porcine epidemic diarrhea virus (PEDV) fusion peptide (FP) on the cell surface, and evaluated their use as killed whole-cell vaccines. The FP sequence is highly conserved across coronaviruses; the six FP core amino acid residues, along with the four adjacent residues upstream and the three residues downstream from the core, are identical between SARS-CoV-2 and PEDV. We tested the efficacy of PEDV FP and SARS-CoV-2 FP vaccines in a PEDV challenge pig model. We demonstrated that both vaccines induced potent anamnestic responses upon virus challenge, potentiated interferon- $\gamma$ responses, reduced viral RNA loads in jejunum tissue, and provided significant protection against clinical disease. However, neither vaccines elicited sterilizing immunity. Since SARS-CoV-2 FP and PEDV FP vaccines provided similar clinical protection, the coronavirus FP could be a target for a broadly protective vaccine using any platform. Importantly, the genome-reduced bacterial surface-expressed vaccine platform, when using a vaccine-appropriate bacterial vector, has potential utility as an inexpensive, readily manufactured, and rapid vaccine platform for other pathogens.

vaccine | genome-reduced bacteria vaccine platform | fusion peptide | porcine epidemic diarrhea virus (PEDV) | SARS-CoV-2

V accine platforms that enable rapid production of new vaccines include many viral vector-, DNA-, and mRNA-based vaccines (reviewed in refs. 1-3). These platforms can produce promising vaccines, but can require dedicated production facilities, employ expensive materials, and can demand logistically challenging cold chains (4).

One of the oldest vaccine technologies is the killed whole-cell vaccine $(\mathrm{KWCV})$ or bacterin. Many developing countries produce KWCVs (for example, pertussis) indigenously. KWCVs are currently licensed to prevent deadly diseases, for example cholera (5), and are produced at large industrial scale for agricultural animals. Several KWCVs have been developed against pathogenic Escherichia coli (reviewed in ref. 6). In one study, mice were immunized subcutaneously with five different pathogenic E. coli KWCVs (7) with no adverse effects noted. In another study, an oral genetically engineered enterotoxigenic E. coli (ETEC) vaccine overexpressing colonization factors was safe in mice (8).
Vaccination specifically with conserved $E$. coli antigens did not alter the gastrointestinal (GI) microbiome $(6,9)$. In human studies, volunteers were immunized orally with a KWCV against ETEC (10) with no adverse effects. An ETEC oral KWCV along with a cholera B toxin subunit adjuvant was studied in children and found to be safe (11). In agriculture the safety of KWCV E. coli vaccines have been reported. A J-5 rough mutant E. coli vaccine was shown to have no adverse effects in neonatal calves (12). Safe and effective $E$. coli KWCVs are currently licensed for several animal diseases, for example J-5 KWCVs for bovine mastitis, such as Enviracor (Zoetis), and J-Vac (Boehringer-Ingelheim) (13). In summary, E. coli KWCVs have an excellent safety record, following both parenteral and nonparenteral administration, using wild-type and genetically modified bacteria and using highly conserved antigens shared by pathogens and commensal strains. KWCVs are attractive for pandemic response, because they are inexpensive, and

\section{Significance}

We report a vaccine platform to express vaccine antigens on the surface of genome-reduced bacteria to enhance vaccine immunogenicity. We demonstrate the utility of this vaccine platform by expressing the highly conserved fusion peptide (FP) of SARS-CoV-2 and porcine epidemic diarrhea virus on the surface of Escherichia coli to produce killed whole-cell bacterial vaccines. The vaccine primes a potent anamnestic response, potentiates interferon- $\gamma$ responses, and provides significant protection in pigs against disease following virus challenge. The FP could be a target for a broadly protective coronavirus vaccine since a betacoronavirus SARS-CoV-2 FP vaccine provided cross-protection against alphacoronavirus porcine epidemic diarrhea virus. When using a vaccine-appropriate bacteria vector, this inexpensive vaccine platform offers the potential for use in developing countries.

Author contributions: X.-J.M. and S.L.Z. designed research; D.L.N.F.M., D.T., H.Y., N.D., V.R., S.M., H.M.M., H.S., B.W., C.L.H., A.H., and T.L. performed research; D.L.N.F.M., D.T., X.-J.M., and S.L.Z. analyzed data; and D.T., X.-J.M., and S.L.Z. wrote the paper.

Reviewers: D.D., Cornell University; and Q.W., The Ohio State University.

Competing interest statement: The University of Virginia and Virginia Polytechnic Institute and State University have filed a provisional patent application covering some of the work described.

This open access article is distributed under Creative Commons Attribution License 4.0 (CC BY).

${ }^{1}$ D.L.N.F.M. and D.T. contributed equally to the work.

${ }^{2}$ To whom correspondence may be addressed. Email: xjmeng@vt.edu or zeichner@ virginia.edu.

This article contains supporting information online at https://www.pnas.org/lookup/suppl/ doi:10.1073/pnas.2025622118/-/DCSupplemental.

Published April 15, 2021. 
facilities currently exist for their manufacture, A description of how 6 million doses of the World Health Organization-prequalified Euvichol oral cholera vaccine were produced in $1 \mathrm{y}$ using a single $100-\mathrm{L}$ bioreactor for $<\$ 1$ per dose further highlights KWCV's advantages (14).

Gram-negative bacterial autotransporters (ATs), proteins that enable bacteria to place proteins into their outer membrane (15-18), have three domains: an $\mathrm{N}$-terminal signal sequence directing transport across the inner membrane; a C-terminal $\beta$-barrel that inserts into the outer membrane, yielding a pore-like structure; and a central passenger domain that transits through the pore, "displaying" the passenger protein to the environment. Sequences encoding a protein of interest can replace the native passenger protein sequence, yielding recombinant ATs that display up to $\sim 2 \times 10^{5}$ foreign proteins on each cell (18). ATs have been used to place vaccine antigens on the bacterial surfaces, elicit immune responses, and in some cases, protective effects (19-23). There are limitations to the use of ATs for antigen expression. Surface expression becomes problematic for antigen sizes $>50 \mathrm{kDa}$, bacteria do not produce antigens with mammalian glycosylation, and antigens with certain secondary structure characteristics, such as extensive disulfide bonds, may be trapped in the periplasmic space. Nevertheless, AT expression of vaccine antigens has held promise, although no licensed vaccines have been produced using the technology, perhaps because antigens expressed on bacterial surfaces via ATs were insufficiently immunogenic.

In this study, we hypothesize that placing recombinant antigens on the surfaces of bacteria lacking a large number of normally present surface proteins would elicit enhanced immune responses against the foreign antigen, therefore potentially informing a new vaccine platform to produce inexpensive vaccines. The Tokyo Metropolitan University Group $(24,25)$ made a systematic set of deletions in the $E$. coli genome and showed that they can delete $29.7 \%$ of the genome, yet retain a viable, albeit slow growing organism $(24,25)$. We used these genome-reduced bacteria to produce KWCVs.

A large number of SARS-CoV-2 candidate vaccines are in development (26), but there are various concerns (27). The approved mRNA-based vaccines are costly to produce and have significant logistic challenges as they require $-20{ }^{\circ} \mathrm{C}$ or $-70{ }^{\circ} \mathrm{C}$ cold-chain transport and storage. Most SARS-CoV-2 vaccines generally target the entire $\mathrm{S}$ protein, tending to elicit strong responses against the immunodominant receptor binding domain (RBD). While S-targeting vaccines are attractive, reports suggest that enhanced immune responses directed against the RBD may be associated with an increased risk of rare inflammatory syndromes associated with COVID-19 (28-30), so exploring alternative subunit vaccine targets in $\mathrm{S}$ is warranted.

Several virus families, including coronaviruses, employ trimeric type I fusion proteins to bind and enter host cells (31). The HIV-1 fusion peptide (FP) has garnered considerable interest as a vaccine target (32). Several potent broadly neutralizing monoclonal antibodies recognize the HIV-1 FP (32-34). For coronaviruses, proteases cleave $\mathrm{S}$ into $\mathrm{S} 1$ and $\mathrm{S} 2$ to activate entry. $\mathrm{S} 1$ binds to its receptor, while $\mathrm{S} 2$ includes an FP that mediates fusion of viral and cellular membranes. Coronavirus FPs consist of 15 to 25 apolar amino acids that reorder the membranes after receptor binding. For SARS-CoV, SARS-CoV-2, and Middle Eastern respiratory syndrome (MERS)-CoV, an $\sim 18$-aa sequence [SFIEDLLFNKVTLADAGF], with a strong homology across different coronaviruses, is typically considered as the FP (35-38). FPs are attractive vaccine candidates because of their minimal sequence variation across the Coronaviridae family (39), with the $93 \%$ of the 39 aa, including those surrounding the FP conserved among all betacoronaviruses and the 39 aa completely conserved among SARS-CoV-2 sequences (40). It would likely be difficult for a virus to evolve so that it would no longer be affected by an immune response directed against the FP (41). For all coronaviruses across four genera, the FP core [IEDLLF] is identical (https:// nextstrain.org). The SARS-CoV-2 FP is one of the sites targeted by preexisting antibodies presumably induced by non-SARS-CoV-2 infection $(38,42,43)$. A human SARS-CoV monoclonal antibody provided protection in a passive challenge infection in a nonhuman primate model (44), suggesting that a vaccine that elicited an analogous immune response would be protective. The binding sites of neutralizing antisera from SARS-CoV-2 patients were also mapped to the FP region (38).

Available animal model systems for SARS-CoV-2 include mice transgenic for ACE2, a hamster model, and nonhuman primate models (45-49). However, none of these models enable the study of a coronavirus in its native host. This may be particularly important because COVID-19 includes many baffling clinical features that involve not only pathology caused directly by the virus, but also host responses triggered by the virus, including coagulopathic (50), vasculitic (51), neurological, and inflammatory phenomena, for example the poorly understood multisystem inflammatory syndrome of children (MIS-C) $(28-30,52)$.

It would be advantageous to test new vaccine concepts in an animal model in which the animal could be challenged with a virus that naturally infects that animal. Porcine epidemic diarrhea virus (PEDV), an alphacoronavirus, causes severe diarrhea worldwide. In 2013, PEDV emerged in the United States, killing millions of pigs and causing immense economic losses to the United States swine industry (53-55). Sequence analysis revealed that the 13-aa residues surrounding and including the core FP sequence [IEDLLF] conserved in all coronaviruses were identical for SARS-CoV-2 and PEDV. This suggested that the efficacy of a SARS-CoV-2 FP vaccine could be tested using a PEDV challenge model in pigs. Pigs are very similar to humans in their genetics, physiology, and anatomy, perhaps the closest model to humans next to nonhuman primates. They have been used as model systems for many infectious disease vaccine studies (reviewed in ref. 56). While the syndromes accompanying PEDV infection in pigs largely involve diarrhea, COVID-19 can include significant GI tract symptoms and pathology, with significant GI viral shedding $(57,58)$.

Here we report a synthetic biology-based KWCV vaccine platform that utilizes ATs to display vaccine antigens on the surfaces of genome-reduced $E$. coli, enabling rapid production of a testable vaccine. As a proof-of-principle for this vaccine platform, we produced KWCVs targeting the FPs of two coronaviruses, SARS-CoV-2 and PEDV, and demonstrated that these vaccines induced potent anamnestic responses upon virus challenge and elicited significant protection against disease in a PEDV challenge pig model, validating the vaccine platform and the use of the coronavirus FP target.

\section{Results}

Establishment of a Vaccine Platform to Express Foreign Antigen on the Surface of Genome-Reduced E. coli. To create a platform for the rapid production of new vaccines, employing killed whole-cell genome-reduced $E$. coli-expressing vaccine antigens on their surfaces, we designed a plasmid, pRAIDA2 (Fig. $1 A$ ), that contains a high copy origin of replication, a kanamycin resistance gene, and an AIDA-I-derived AT surface-expression cassette with a rhamnoseinducible promoter. After the AT amino terminal signal sequence, pRAIDA2 has a cloning site flanked by type IIS BbsI restriction sites, enabling "scarless" cloning into the expression cassette. The parental version of the plasmid includes a sequence encoding an influenza virus HA immunotag as stuffer, flanked by a trypsin cleavage site, to enable confirmation and evaluation of surface expression (Fig. 1A). Fig. $1 B$ illustrates a pathway for the rapid production of synthetic biology-mediated candidate vaccines using pRAIDA2, or similar systems, and genome-reduced bacteria.

To determine if the vaccine antigens expressed on the surface of the bacteria would be more visible to the immune system if expressed on bacteria with a large number of the surface protein 
A

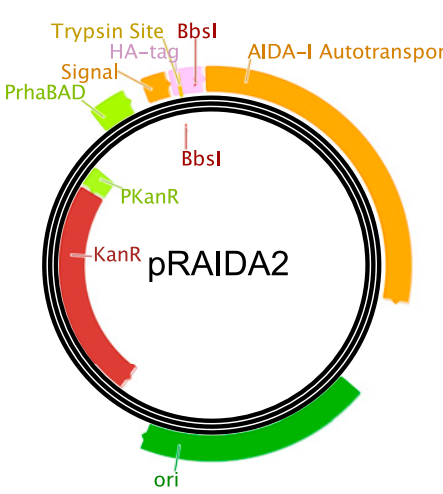

Vaccine Production Schematic

Identify Sequence/Codon Optimize

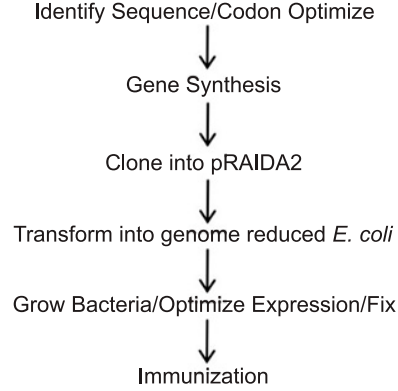

Fig. 1. Vaccine platform design and implementation. (A) Map of synthetic plasmid pRAIDA2. Design features include a high copy origin of replication, a kanamycin-resistance marker, and an AIDA-I AT expression cassette under the control of a rhamnose-inducible promoter. The expression cassette has a cloning site flanked by BbsI type IIS restriction sites. In its original, parental version, pRAIDA2 expresses an influenza virus HA immunotag. (B) A schematic diagram of the general process of candidate vaccine production using pRAIDA2 and genome-reduced bacteria.

genes deleted, we first examined the collection of systematically deleted E. coli strains produced by the Tokyo Metropolitan University Group $(24,25)$ and identified genes encoding proteins with an imputed location on the surface of the cell (Fig. $2 A$ and Dataset S1). The strain with the largest amount of genomes deleted in the collection included deletions in almost 200 genes encoding proteins imputed to be on the cell surface.

Enhanced Antibody Binding to an Antigen Expressed on the Surface of Genome-Reduced $\boldsymbol{E}$. coli. We tested the ability of bacteria with different amounts of genome deletions that had been transformed with pRAIDA2 which, in its parental versions, expresses an HA immunotag via the AIDA-I AT on the bacterial surface, to bind an anti-HA monoclonal antibody (Fig. $2 B$ ). We found that binding of the monoclonal antibody to the bacteria increased as a function of genome reduction (Fig. $2 B$ ).

To demonstrate that the recombinant HA immunotag was properly expressed on the surface of the bacterial strains and to eliminate the possibility that the increased binding observed in the flow cytometry experiments was due to quantitative differences in the amount of HA expressed in the bacteria, we conducted trypsinization-immunoblot experiments in which we induced expression of the HA immunotag with rhamnose, then either did or did not subject the bacteria to trypsin treatment prior to making protein extracts for immunoblotting (Fig. $2 C$ ). We found that the HA immunotag was expressed on all the tested mutants. The amount of HA protein expressed in the different strains was approximately equal (varying by $<16 \%$ from the most abundant to least abundant, normalized to DNAK as a loading control, with the ME5125 having the lowest normalized protein amount), indicating that the increased binding seen with the highly deleted strains was not the result of quantitative differences in protein expression. The HA immunotag was accessible to trypsinization in all the strains, providing further evidence that the HA immunotag expressed via pRAIDA2 was located on the surface of the bacteria.

Successful Surface Expression of SARS-CoV-2 FP and PEDV FP on GenomeReduced E. coli, Production and Characterization of Candidate Vaccines. To test the ability of the genome-reduced $E$. coli surface expression to yield a useful vaccine, we selected the conserved FP region of the coronavirus $\mathrm{S}$ protein as the target (Fig. 3A) (41). An FPtargeting vaccine would be relatively resistant to viral evolution or mutation. Monoclonal antibodies directed against the SARSCoV FP were neutralizing and protective in passive challenge experiments (44). In addition to the SARS-CoV-2 FP, we also produced, in parallel, a PEDV FP vaccine, and tested them in a PEDV native virus challenge pig model. For our test vaccine antigens, we included the basic 18-aa FP, plus flanking sequence that had been mapped as being included in the binding sites for neutralizing and disease-modifying-associated sera and neutralizing monoclonal antibodies against SARS-CoV and SARS-CoV-2 (38, 42-44), for the SARS-CoV-2 construct, and the corresponding amino acids for PEDV, to include a total of 23 aa (Fig. $3 A$ ).

We initially conceived of the SARS-CoV-2 FP vaccine as a negative control to assess protection elicited by the PEDV FP vaccine, given that PEDV is an alphacoronavirus and SARS-CoV-2 is a betacoronavirus. However, sequence alignment of the SARS-CoV-2 and PEDV FP sequences used in this study (Fig. 3A) revealed that the 13-aa residues surrounding the core FP sequence, including the six core residues, four adjacent residues immediately upstream from the core, and three adjacent residues immediately downstream from the core are identical between SARS-CoV-2 FP and PEDV FP, suggesting that the efficacy of a SARS-CoV-2 FP vaccine may be tested by using the PEDV challenge pig model as well. We also produced rabbit polyclonal antibodies directed against the PEDV and SARS-CoV-2 FPs, respectively. We transformed pRAIDA2-SARS-CoV-2 FP and pRAIDA2-PEDV FP into the wild-type E. coli strain ME5000 (0\% genome-deleted) and strain ME5125 (29.7\% genome-deleted) and conducted flow cytometry

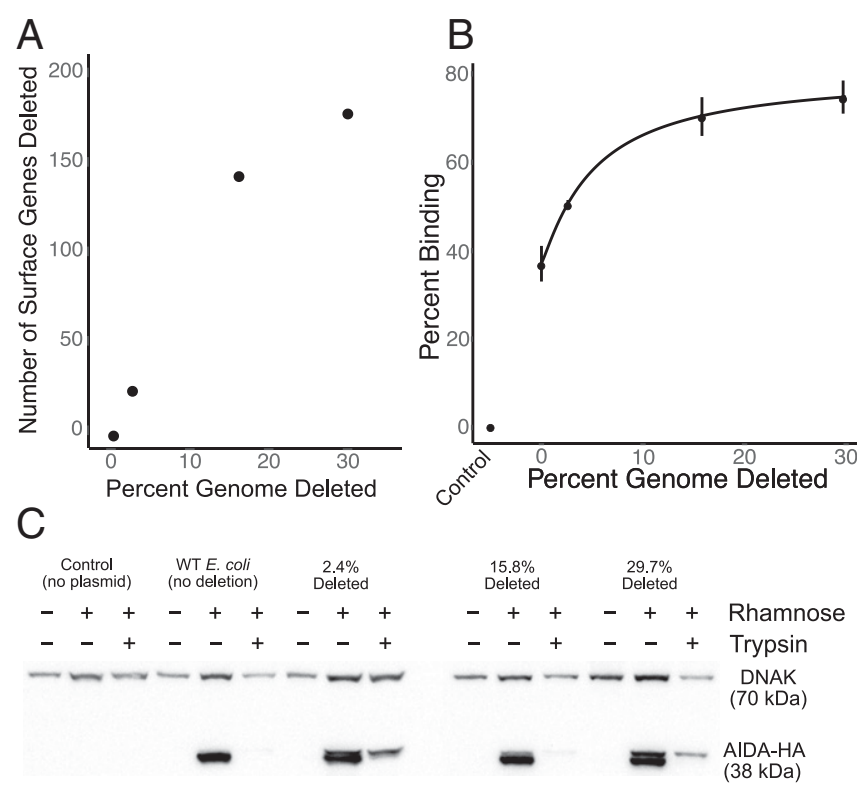

Fig. 2. Genes with imputed locations on the surface of the genomereduced bacteria and increased binding of a monoclonal antibody against a recombinant antigen expressed on the surfaces of genome-reduced bacteria transformed with pRAIDA2. (A) Genes removed with imputed locations on the surface of $E$. coli strains ME5000, ME5110, ME5119, and ME5125 as a function of percent genome deleted. $(B)$ Binding of a commercial anti-HA monoclonal antibody to the surfaces of the genome-reduced bacteria as a function of percent genome deleted. (C) Immunoblots of extracts from the wild-type and genome-reduced bacteria, transformed with pRAIDA2, and with expression of the HA immunotag with or without rhamnose induction (indicated at the top). Also indicated is whether the bacteria were pretreated with trypsin prior to protein extraction. Rhamnose induces expression of the HA immunotag expressed via the AT expression cassette. Trypsin treatment removes the HA immunotag from the bacterial surface. DNAK was probed as a loading control. The normalized amount of AIDA-HA differed by $<16 \%$ from strain to strain, with the ME5125 $29.7 \%$ deleted strain expressing the least normalized AIDA-HA. 

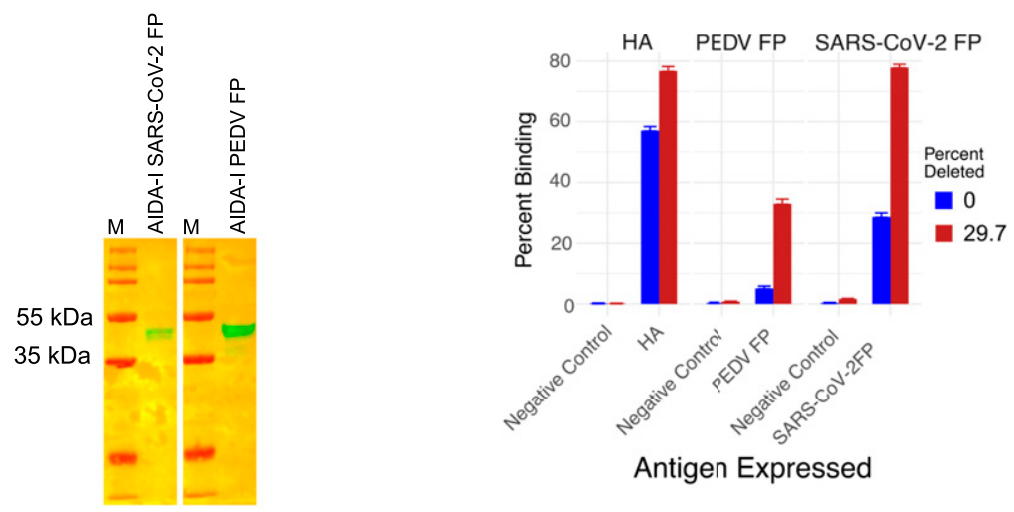

D
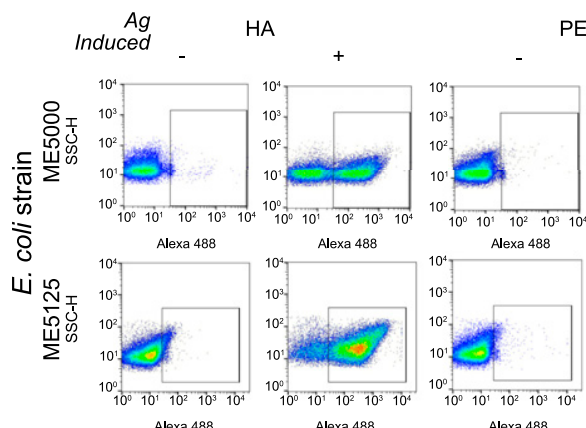

PEDV FP
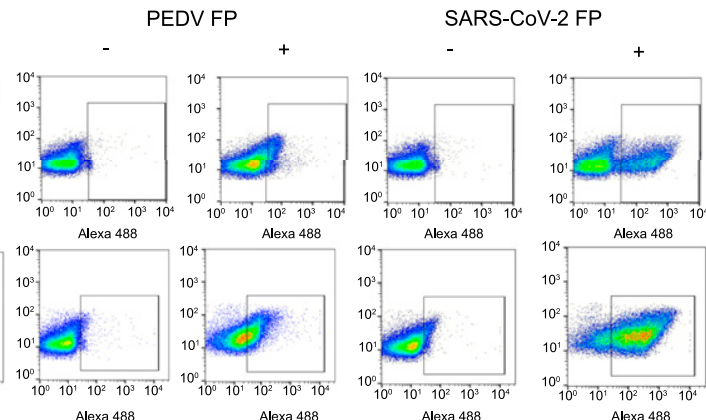

Fig. 3. FPs from PEDV and SARS-CoV-2 and their surface expression in genome-reduced bacteria. (A) Alignment of FPs from PEDV and SARS-CoV-2 used in the candidate vaccines. Uppercase letters indicate the 13-aa residues that are identical between the PEDV FP and SARS-CoV-2 FP. Uppercase letters in bold indicate FP core sequence that is conserved among coronavirus sequences. (B) Immunoblots confirming expression of the FPs in extracts of the bacteria transformed with pRAIDA2-PEDV, or pRAIDA2-SARS-CoV-2, probed using the anti-PEDV FP or anti-SARS-CoV-2 FP rabbit polyclonal antibodies. (C) Summary of pooled repeat flow cytometry experiments. The antigens are consistently expressed on the bacteria, and binding to the genome-reduced bacteria is consistently greater than to the wild-type parental bacteria. (D) An example of a flow cytometry experiment conducted on wild-type (ME5000) and 29.7\% genome-reduced $E$. coli (ME5125), transformed with parental pRIADA2 expressing the HA immunotag (HA), pRAIDA2-PEDV (PEDV FP), or pRAIDA2SARS-CoV-2 (SARS-CoV-2 FP). Bacteria were treated with (+) or without $(-)$ rhamnose to induce expression of the FP cloned into the pRAIDA2 expression cassette. Cells were stained with a commercial anti-HA monoclonal antibody, or with rabbit polyclonal anti-PEDV FP or rabbit polyclonal anti-SARS-CoV-2 FP, and then with the appropriate Alexa 486-conjugated secondary antibodies. The results revealed that the HA immunotag and the FPs are expressed on the surface of the bacteria, and binding is enhanced when the proteins are expressed on the surface of the genome-reduced bacteria.

experiments using the rabbit anti-FP antibodies to demonstrate that the FP antigens were successfully expressed on the bacteria. We verified the expression of the FP-AT recombinant protein by immunoblot (Fig. $3 B$ ). We conducted repeated binding experiments, assessing binding of a rabbit polyclonal anti-FP antibody to the surfaces of the bacteria expressing the FP by flow cytometry (two to four experiments summarized in Fig. $3 C$ ), and the flow cytometry dot plots of an additional experiment is shown in Fig. $3 D$. The experiments showed that the FP antigens are present on the surfaces of the genome-reduced $E$. coli, and also showed that, for both these FP antigens, expression on the highly deleted ME5125 strain yielded substantially increased binding (Fig. $3 C$ and $D$ ), as we had similarly observed for the surface-expressed HA immunotag shown in Fig. $2 B$.

To produce and characterize the SARS-CoV-2 FP and PEDV FP candidate vaccines, we grew the highly deleted $(29.7 \%)$ ME5125 E. coli strain transformed with pRAIDA2-SARS-CoV-2 and pRAIDA2-PEDV, respectively, induced expression with rhamnose, and then inactivated the bacteria with formalin. Expression of
FP antigens on the bacterial cell vaccines was verified by flow cytometry, and the amount of FP expression was quantitated by immunoblot, using serial dilutions of DNAK as the quantitation standard.

SARS-CoV-2 FP and PEDV FP Vaccines Induce Low Anti-FP Humoral Response but Potent Anamnestic Responses after Virus Challenge. To test the ability of the killed whole-cell genome-reduced bacterial vaccines expressing the FPs to elicit a useful immune response, we vaccinated pigs intramuscularly with the KWCVs expressing the SARS-CoV-2 FP or PEDV FP or control bacteria not expressing a coronavirus FP on day 0 , boosted at day 21 , and then challenged with infectious PEDV orally at day 35 . Fecal samples and swabs were collected daily and blood was collected weekly. The production of antibodies recognizing the FPs was examined by ELISAs (Fig. $4 A$ and $B$ ). We found that vaccination with the SARS-CoV-2 FP or PEDV FP vaccines did not elicit a strong anti-FP response by week 5, 2 wk after boosting, although we noticed that the SARS-CoV-2 FP vaccine elicited a low, but statistically 
significant response against the PEDV FP $(P<0.05$, Wilcoxon rank sum test). The immune response against the SARS-CoV-2 FP is not statistically significant $(P=0.25)$. Importantly, both vaccines primed the pigs for a potent anamnestic response against either FP after the pigs were infected with PEDV. At the end of the challenge experiment, anti-FP ELISA values for the pigs vaccinated with the FP vaccines were all significantly $(P<0.05$, Wilcoxon rank sum test) higher than values for the pigs vaccinated with the control vaccine.

At necropsy ( $7 \mathrm{~d}$ postchallenge, dpc), anti-PEDV neutralizing antibodies were detected in 1:40 diluted sera from five of seven PEDV FP-vaccinated pigs, two of seven SARS-CoV-2 FP-vaccinated pigs, and in two of seven control pigs challenged with PEDV. The neutralizing antibody (NA) responses in the two animals from the control group are likely induced by the challenge PEDV. However, neutralizing antibody against PEDV at challenge day, and against SARS-CoV2 S pseudovirus at challenge or necropsy day, was not detected in vaccinated or control group of pigs.

Vaccines Potentiate Interferon- $\gamma$ Response in Vaccinated Pigs. The level of interferon (IFN)- $\gamma$ in pig serum samples were tested and compared between the vaccinated groups and control group at each time point (Fig. 4C). There were significant differences at 5 wk postvaccination (wpv, $P<0.05$ ) and $1 \mathrm{wk}$ postchallenge (wpc,
$P<0.05)$. The results showed that the serum IFN- $\gamma$ levels significantly increased $2 \mathrm{wk}$ after the vaccine booster dose (5 wpv) and $1 \mathrm{wk}$ postchallenge ( $1 \mathrm{wpc}$ or $6 \mathrm{wpv}$ ) in the vaccinated groups as compared to the control group. The IFN- $\gamma$ levels at $1 \mathrm{wpv}$ and 3 wpv are similar, but the IFN- $\gamma$ level at $5 \mathrm{wpv}$ (i.e., 2 wk after booster) increases in vaccinated groups, suggesting that the vaccine prime dose likely has activated T cells, and the booster dose further amplifies the activation. The results suggested that the FP vaccines potentiate IFN- $\gamma$ response in vaccinated animals.

Vaccines Reduced Clinical Signs and Pathological Lesions in Pigs after PEDV Challenge. The efficacy of the two genome-reduced bacteriavectored surface-expression FP vaccine candidates (SARS-CoV-2 FP and PEDV FP) was evaluated in a pig vaccination and challenge study against PEDV (strain 2013 Colorado). Since severe disease is usually found in younger PEDV-infected piglets while the pigs used in this study were $\sim 10$-wk-old at the time of virus challenge, a higher dose of PEDV $\left(3.0 \times 10^{5.0} \mathrm{TCID}_{50}\right.$ per pig $)$ was used to challenge the pigs. Clinical observations were conducted for the immediate 2 to $4 \mathrm{~h}$ after vaccination and daily thereafter, and included an assessment of the pig's body condition and stool/diarrhea output. A few vaccinated pigs exhibited lethargy, labored breathing, and vomiting immediately after vaccination, which resolved shortly after intramuscular administration of diphenhydramine.
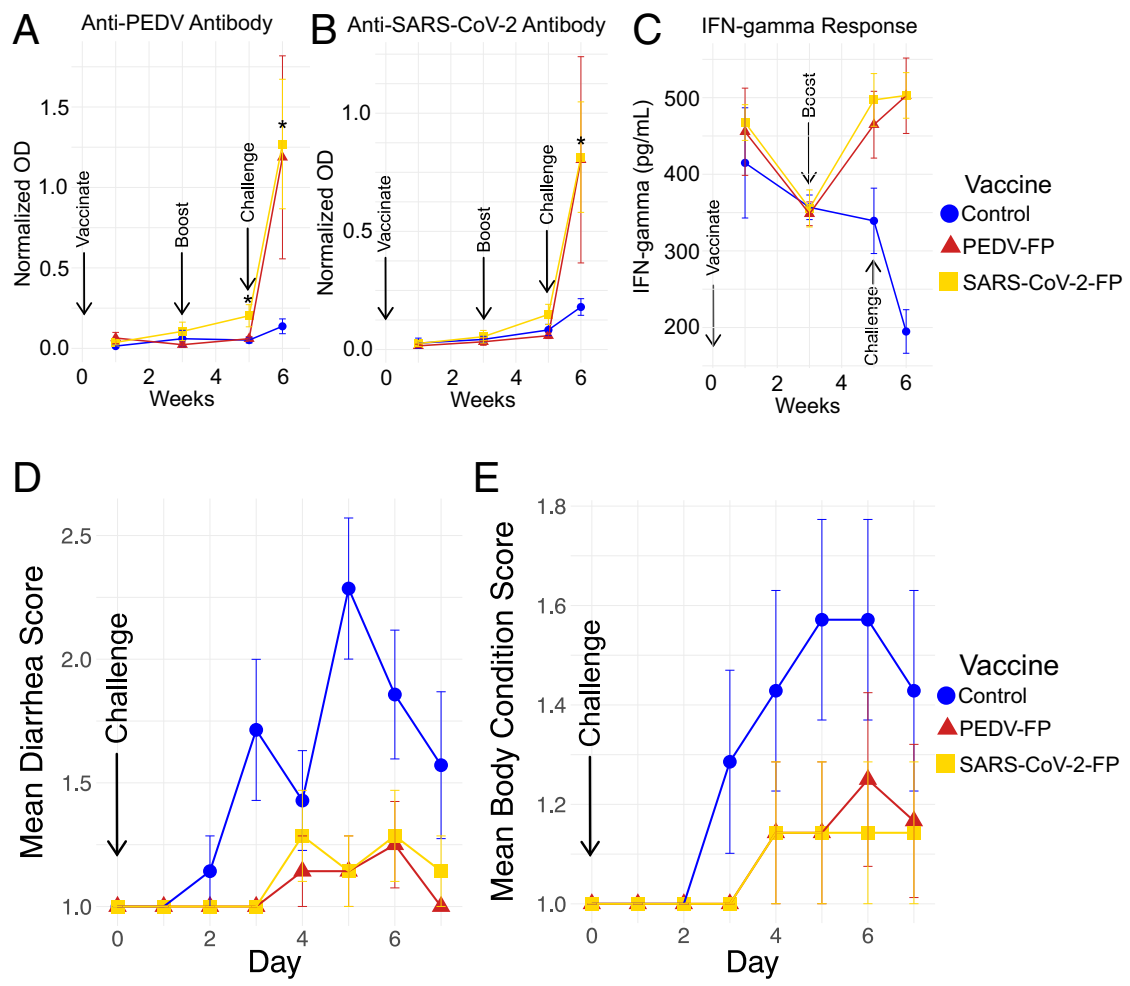

Fig. 4. Pig humoral and IFN- $\gamma$ responses after vaccination with killed whole-cell genome-reduced vaccines expressing FPs using pRAIDA2 and pig clinical responses after vaccination and PEDV challenge. ( $A$ and $B$ ) Pig humoral immune responses against the PEDV FP $(A)$ or SARS-CoV-2 FP $(B)$ following vaccination with the PEDV FP or SARS-CoV-2 FP vaccine and subsequent virus challenge. Normalized OD (sample OD - negative control OD/positive control OD - negative control OD). At challenge day, there was a small, but statistically significant increase in anti-PEDV FP antibody in the pigs vaccinated with the SARS-CoV-2 FP vaccine $(P<0.05$, Wilcoxon rank sum test), while not significant against the SARS-CoV-2 FP itself $(P=0.25)$ in pigs vaccinated with the SARS-CoV-2 FP vaccine. At necropsy day, there was a strong and statistically significant $(P<0.05$, Wilcoxon rank sum test) anamnestic response against both PEDV FP and SARS-CoV-2 FP in pigs vaccinated with PEDV and SARS-CoV-2 FP vaccines, respectively. (C) IFN- $\gamma$ responses in serum samples of vaccinated and control pigs. There were significant differences at $5 \mathrm{wpv}(P<0.05)$ and $1 \mathrm{wpc}(P<0.05)$ between the vaccinated groups and control. $(D)$ Diarrhea scores following PEDV challenge. $(E)$ Body condition scores following PEDV challenge. Both PEDV FP and SARS-CoV-2 FP vaccines provided substantial and highly statistically significant protection against adverse clinical effects observed following the PEDV challenge infection $(P<0.01$ for all groups, Friedman rank sum test, comparing each vaccinated group to the control for both diarrhea and body condition scores). Diarrhea scores range from 1 to 3 , where 1 is normal to pasty feces, 2 is semiliquid diarrhea with some solid content, and 3 is liquid diarrhea with no solid content. Body condition scores range from 1 to 3 , where 1 is undetectable spinous processes and hook bones, 2 is spinous processes and hook bones were slightly felt, and 3 is spinous processes and hook bones were easily felt and visible. 


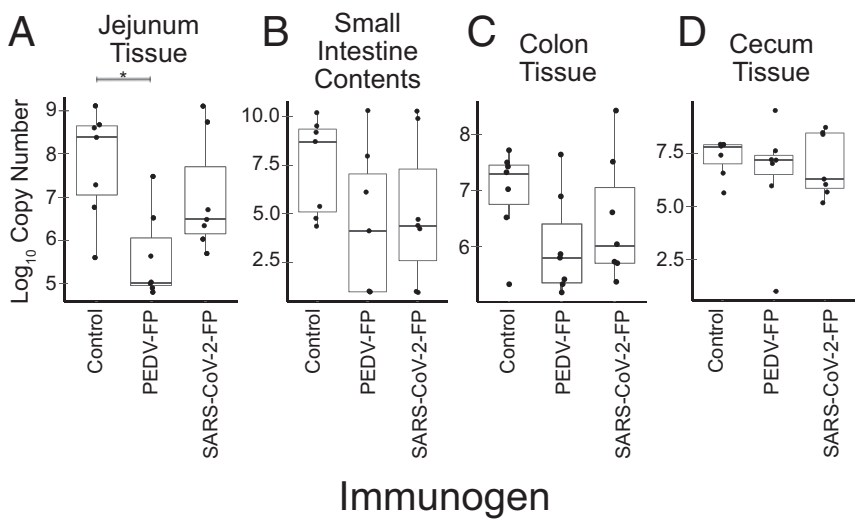

Fig. 5. Pig intestinal compartment viral RNA loads. Effects of vaccination on the viral RNA loads in the jejunum tissue $(A)$, small intestine contents from necropsy $(B)$, colon tissue $(C)$, and cecum tissue $(D)$. There was a significant difference in viral RNA loads in the comparison of control vs. PEDV vaccine in the jejunum tissue ( $P=0.01$, Kruskal-Wallis test). The $P$ values for comparison of control vs. PEDV vaccine in the small intestine content collected at necropsy was $P=0.158$, and in the colon tissue $P=0.11$. Similar to PEDV FP, the SARS-CoV-2 FP-vaccinated pigs also have numerically lower, but not statistically significant, PEDV loads compared to control pigs.

The PEDV-related clinical signs of diarrhea and body condition were scored (Fig. $4 D$ and $E$ ). At $2 \mathrm{dpc}$, one pig in the unvaccinated control group began to show clinical signs of diarrhea, and in total, six of the seven pigs developed diarrhea during the course of study. Most of the pigs developed marasmus along with diarrhea. In both vaccinated groups, most of pigs remained healthy and only one to two pigs showed mild diarrhea and marasmus during the study. Friedman rank sum tests comparing the vaccinated groups after day 3 to the control for both diarrhea and body condition scores were highly significant ( $P<0.01$ for all groups), suggesting that the vaccines significantly reduced the clinical signs after virus challenge.

Vaccines Decreased Viral RNA Loads in the Jejunum Tissue of Pigs after PEDV Challenge. PEDV RNA was detected in pigs starting at $2 \mathrm{dpc}$; however, there was no significant difference in viral RNA loads in daily fecal swab materials, although swabs may not accurately sample fecal virus, due to sampling inconsistency or imperfect dispersion of virus in feces. Intestine tissues (jejunum, colon, cecum) and small intestinal contents were collected during necropsy $(7 \mathrm{dpc})$ to more accurately quantify the viral RNA loads in each pig. There was a significant difference in viral RNA loads in the comparison of control vs. PEDV vaccine in the jejunum tissue (Fig. 5A) $(P=0.01$, Kruskal-Wallis test). The comparison of control vs. PEDV vaccine, however, was not statistically significant in the small intestine content (Fig. $5 B)(P=0.158)$, in colon tissue (Fig. $5 C)(P=0.11)$, or in cecum tissue (Fig. $5 D)$. Similar to PEDV FP, the SARS-CoV-2 FP vaccinated pigs also have numerically lower, but not statistically significant, viral loads compared to control pigs.

We also collected, scored, and analyzed clinical indicators of disease at necropsy by determining intestine content scores and histopathological scores of villous blunting. For the intestine contents score at necropsy (Fig. $6 A$ ), both vaccinated groups showed lower scores than the control group, and the difference between SARS-CoV-2 FP group and control group was borderline statistically significant $(P=0.055$, Kruskal-Wallis test). The difference between the PEDV FP group and the control group was not statistically significant $(P=0.08)$. For the histopathological lesion score (Fig. $6 B)$, the mean ratios of villous length to crypt depth $(\mathrm{V}: \mathrm{C})$ of jejunum tissues from both vaccine groups had higher values (more healthy jejunum) than the control group, although the difference was not significant for either the PEDV FP vaccine $(P=0.20$, KruskalWallis) or the SARS-CoV-2 FP vaccine $(P=0.27)$, in part due to the dispersion of the values. In all groups of pigs, only two pigs in the unvaccinated control group developed either thin walled or gas-distended jejunum, while other pigs did not show any gross lesion in intestines. The pathological data suggested that the vaccinated pigs had less severe lesions associated with PEDV infection. The overall gross and histological lesions of pigs in this study were mild because the 10 -wk-old pigs were less vulnerable to PEDV-induced disease.

\section{Discussion}

There is encouraging evidence that vaccines against SARS-CoV-2 consisting of the entire $\mathrm{S}$, or the RBD, can provide excellent protection against clinically significant disease, although the ability of the vaccines to protect against infection per se has not yet been well established (59). However, there are also reports that patients who recover from mild COVID-19 can be reinfected by a different SARS-CoV-2 strain $(60,61)$, and that this can happen in patients who have antibodies against the $\mathrm{S} 1$ region of $\mathrm{S}$ and the RBD. There are reports of SARS-CoV-2 with mutations with enhanced transmissibility (62-66), and reports of mutations, some in RBD, suggesting that currently used vaccines may have decreased effectiveness (67-69). It may be helpful to include extremely well-conserved but less immunodominant antigens, such as the FP antigen we used in this study, in future SARS-CoV-2 vaccines, employing whatever vaccine platform.

It may also be helpful to further explore additional vaccine platforms that yield vaccines quickly and inexpensively, and can be stored and transported easily for globally appropriate use. We embarked on this project to explore whether expressing foreign antigens on the surface of genome-reduced gram-negative bacteria using gram-negative AT expression systems could allow antigens to interact more effectively with the immune system, and so yield a synthetic biology-based new vaccine platform to rapidly produce inexpensive vaccines. While the candidate vaccines we produced in this initial proof-of-concept study did not elicit strong neutralizing humoral immune responses using the arbitrarily chosen dose, immunization route, and vaccination schedule, we observed
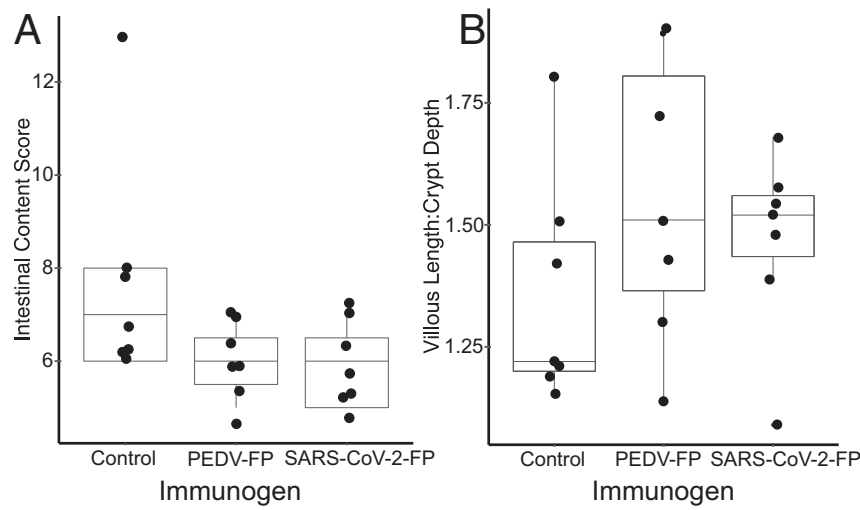

Fig. 6. Pig histological lesion and intestinal content clinical scoring at nec ropsy after vaccination and challenge with PEDV. $(A)$ The Intestinal Content Score recorded at necropsy. The difference between SARS-CoV-2 FP group and control group was borderline statistically significant $(P=0.055$, Kruskal-Wallis test). The difference between the PEDV FP group and the control group was not statistically significant $(P=0.08)$. (B) Histopathological scoring of intestinal villous length to crypt depth. The mean ratios of $\mathrm{V}: \mathrm{C}$ of jejunum tissues from both vaccine groups had higher values (more healthy jejunum) than control group, although the difference were not significant, for either the PEDV FP vaccine ( $P=0.20$, Kruskal-Wallis) or the SARS-CoV-2 FP vaccine $(P=0.27)$, in part due to the dispersion of the values. 
significant protection against severe disease in the PEDV pig challenge model. We demonstrated a strong anamnestic response upon virus challenge, and evidence of differences in clinical correlates of disease and virus production. The FP vaccine we used was also encouraging in that it provided protection against viral pathologic effects, since a previously produced dendritic celltargeting PEDV S protein vaccine given to sows, reduced viral shedding in their experimentally infected piglets, but was associated with enhanced gross pathologic lesions (70). SARS-CoV-2 is predominantly a respiratory disease; however, it does have significant GI symptomatology and substantial amount of virus is shed in feces $(71,72)$. GI infection with SARS-CoV-2 can cause pneumonia and GI dysfunction in rhesus macaques (58). PEDV causes predominantly a GI disorder. While the two diseases have distinct characteristics, as pandemic coronaviruses transmitted across a mucosal barrier the two diseases have useful parallels in GI symptoms, and the fact that vaccine prevention of PEDV can be studied in its native host enhances the model's realism.

It is reasonable to consider that with optimization of the dose, immunization schedule, and route-notably the use of vaccination via oral or intranasal mucosal compartments, additional reduced genome, or incorporation of appropriate adjuvants in the future-vaccines made using this genome-reduced bacterial surface-expression vaccine platform may elicit better immune responses and may be valuable for rapid responses to future pandemic viral diseases.

Other highly conserved antigens could also be expressed using the platform, or vaccines targeting multiple antigens could yield a better immune response. Our vaccine platform employed $E$. coli with large, but essentially arbitrary mutations. More targeted mutations limited to surface-expressed genes, with fewer effects on bacterial growth may improve production. Deletion of even more, nonessential surface-expressed proteins may yield better immunogenicity. Additional derivatives of the genome-reduced bacteria (minicells, outer membrane vesicles) could also enhance immune responses. Further research, which is beyond the scope of this present study, is warranted to address these questions.

There are theoretical safety concerns regarding the vaccine platform. For example, it is possible that an immune response elicited by genome-reduced $E$. coli against the bacteria themselves might have harmful effects on the host or host microbiome. However, we did not observe significant adverse effects in the pigs vaccinated with the vaccines or with the control bacteria not expressing a vaccine antigen during the $6 \mathrm{wk}$ before virus challenge. A number of preclinical (6-8) and human clinical studies $(10,11)$ with various $E$. coli KWCVs failed to show adverse effects from the vaccinations, including specific effects on the microbiome (9), and the clinical experience with approved veterinary $E$. coli $\mathrm{KWCV}$ s suggest that a genome-reduced $E$. col KWCV would unlikely have significant adverse effects. Nevertheless, long-term safety studies, beyond the scope of this study, would clearly be needed prior to the clinical use of any genomereduced $E$. coli vaccine. It may be advantageous to use, instead of a commensal genome-reduced $E$. coli as the vaccine vector, a genome-reduced pathogenic $E$. coli strain, or even a genomereduced Salmonella Typhi, Bordetella pertussis, or Vibrio cholera as a bacterial vaccine vector, so that any immunity elicited against the vector would likely be beneficial. There exist numerous licensed human KWCVs employing $B$. pertussis, and $V$. cholera, and Salmonella human and animal vaccines with good safety profiles, although older parenteral killed whole-cell typhoid vaccines were reactogenic (73).

Employing this vaccine platform, we found that the FP may be a potential target for a universal coronavirus vaccine. We selected the FP because it is extremely well-conserved among coronaviruses, and because the FP of other viruses with type 1 viral fusion proteins have also been the target of considerable vaccine development efforts. The finding that the SARS-CoV-2 FP and PEDV
FP vaccines exhibited similar protective effect in the PEDV challenge pig model system was welcome, but not surprising, since the six FP core amino acid residues along with the four adjacent residues upstream the core and the adjacent three residues downstream the core are identical between PEDV and SARS-CoV-2. The core 6-aa residues are identical in FPs of all other coronaviruses. The finding that the FP vaccines from different genera of coronaviruses (alpha- and betacoronaviruses) were similar in protective effect suggests that the FP may be a useful target for development of a broad coronavirus vaccine, and that it may be helpful to include an FP-specific antigen in future, next-generation of SARS-CoV-2 vaccines, since SARS-CoV-2 evolution is becoming problematic.

Finally, while we did not find that the two candidate vaccines, used at the dose, vaccination route, and schedule we describe here, elicited sterilizing immunity, they did elicit a potent anamnestic response, a significantly higher IFN- $\gamma$ response, and provided significant protection against clinical disease. Some licensed animal coronavirus vaccines protect against clinical diseases but not against infection (74). For example, canine coronavirus vaccines protect dogs from disease but not from infection (75). A recent passive immunization study in nonhuman primates suggested that high-level neutralizing humoral immunity may not be essential for protection against SARS-CoV-2 disease (76). A very inexpensive, easy to manufacture vaccine with forgiving supply chain requirements and logistical challenges that does not elicit sterilizing immunity, but still helps protect against clinically significant disease, and is resistant to viral evolution or mutation may yet be helpful in a global context. Given estimates that sufficient courses of the current COVID-19 vaccines may not be available to vaccinate much of the global population in developing countries until substantially later than the industrialized countries (77), and that the cost of many of these current vaccines and their requirement for very low temperature storage and transport may present a challenge for the poorer countries in the world, additional, globally targeted SARS-CoV-2 vaccines may prove helpful.

\section{Materials and Methods}

Plasmid Synthesis. The plasmid pRAIDA2, which contains a high copy origin of replication, a kanamycin-resistance gene, and a slightly modified AIDA-I AT surface expression cassette under the control of a rhamnose inducible promoter, was synthesized by GeneWiz (Fig. 1A). The expression cassette has a cloning site with type IIS Bbsl restriction sites to enable "scarless" cloning. The stuffer in the parental version of the plasmid encodes an influenza HA immunotag to enable verification of expression. The sequence of pRAIDA2 has been deposited into GenBank with accession number MW383928. Plasmids were prepared using Qiagen Plasmid Mini Prep kit, quantitated, and assessed for quality spectrophotometrically. The construction of vaccines employing a synthetic gene cloned into the pRAIDA2 expression cassette and then expressed on genome-reduced bacteria is schematically illustrated in Fig. $1 B$.

Bacteria. E. coli strains, including the parental strain and highly genomedeleted strains, with varying amounts of bacterial genome deletion, were a gift of J. Kato (Tokyo Metropolitan University, Tokyo) $(24,25)$, obtained through the National Bioresource Project, E. coli Strain Office, National Institute of Genetics, Japan. The E. coli strains used in this study, MG1655 derivatives, include ME 5000 (wild-type, with $0 \%$ of the genome deleted), ME 5010 (2.4\% deleted), ME 5119 (15.8\% deleted), and ME 5125 (29.7\% deleted). E. coli strains were grown in LB media and on LB agar plates with appropriate antibiotics. For molecular cloning work, chemically competent E. coli DH5a (ThermoFisher) were used for transformations per manufacturer's instructions.

To prepare electrocompetent cells, bacteria were grown in a shaker overnight at $37{ }^{\circ} \mathrm{C}$, inoculated from overnight culture and grown to log phase. Cells were collected by centrifugation and washed with ice-cold phosphate-buffered saline (PBS)-10\% glycerol, resuspended in ice-cold water-10\% glycerol, and transformed via electroporation with the pRAIDA2derived plasmids expressing the SARS-CoV-2 FP or PEDV FP, respectively. Electroporation was conducted in $0.1-\mathrm{cm}$ electroporation cuvettes with the Gene Pulser Xcell electroporation system (Bio-Rad) and pulsed at settings: $1,800 \mathrm{~V}, 25 \mu \mathrm{F}$, and $200 \Omega$. Electroporated cells were transferred to $1.5-\mathrm{mL}$ 
microcentrifuge tubes with $1 \mathrm{~mL}$ of SOC media (Life Technologies), and grown in an orbital shaker $(80 \mathrm{rpm})$ at $37{ }^{\circ} \mathrm{C}$ for $1 \mathrm{~h}$ before plating on LB agar plates containing the appropriate antibiotic.

Analysis of Genes with Imputed Expression on the Bacterial Surface. We examined the lists of genes included in the genome-reduced $E$. coli strains used in this study $(24,25)$. The names of the genes in each deletion were gathered from the Japan National Institute of Genetics National BioResource Project E. coli Strain website (https://shigen.nig.ac.jp/ecoli/strain). Most of the information, including the gene name, protein name, its product, location, function, gene ontology, and other notes about the genes, were retrieved from the National BioResource Project of Japan (https://shigen.nig.ac.jp/ ecoli/strain/resource/longDeletion/lddTablelnfo). We also queried the Uniprot and Ecocyc databases (https://www.uniprot.org/; https://ecocyc.org/). This information is listed in the supporting information as Dataset S1, in which the genes are listed, along with the mutants they have been deleted from and their imputed location in the bacterial cell. The data were analyzed in $\mathrm{R}$ and plotted. The number of surface genes deleted vs. percent genome deleted is shown in Fig. $2 A$.

Design, Synthesis, and Cloning of FP Coding Sequences from SARS-CoV-2 and PEDV into pRAIDA2. We synthesized $E$. coli codon-optimized DNAs (Blue Heron) encoding the SARS-CoV-2 FP: AACCACGTCTTCACCGAGCAAACCGAGCAAACG CAGCTTCATCGAGGATCTGCTGTTCAACAAGGTGACGCTGGCCGATGCCGGTTTTGGTGGCGGCAGAAGACTTGTGT and PEDV FP: AACCACGTCTTCAGGCCGCGTTGTTCAGAAACGCAGCTTCATCGAGGATCTGCTGTTCAACAAGGTGGTGACCAATGGTCTGGGCACCGGTGGCGGCAGAAGACTTGTGT.

The FP-encoding DNAs were digested with BbS I (New England Biolabs), gel-purified, and ligated into Bbs l-digested pRAIDA2, transformed into chemically competent $\mathrm{DH} 5 \mathrm{a}$, and plated on LB agar containing kanamycin.

Production of KWCV. We used overnight cultures to start 50-mL cultures in LB broth with the appropriate antibiotics, incubated in a shaker at $210 \mathrm{rpm}$, at $37{ }^{\circ} \mathrm{C}$, overnight. The next day, the $50-\mathrm{mL}$ overnight cultures were diluted $1: 10$ in LB broth with antibiotic, and cells were grown to midlog-phase growth $\left(\mathrm{OD}_{600} \sim 0.5\right.$ to 0.6$)$. We induced recombinant protein expression with L-rhamnose (SigmaAldrich), added to a 5-mM final concentration, and incubated the bacteria for an additional $2 \mathrm{~h}$ at $37^{\circ} \mathrm{C}$, in a shaker at $210 \mathrm{rpm}$. The bacteria were collected by centrifugation at $5,000 \times g$ for $20 \mathrm{~min}$ at $4{ }^{\circ} \mathrm{C}$. For flow cytometry and vaccine production, the pellet was resuspended in $10 \mathrm{~mL}$ of Hank's Balanced Salt Solutions (HBSS) with $0.2 \%$ formalin (SigmaAldrich), the cells were incubated at $37{ }^{\circ} \mathrm{C}$ for $1 \mathrm{~h}$, shaking at $180 \mathrm{rpm}$. The bacteria were resuspended in $1 \times \mathrm{PBS}$ with $20 \%$ glycerol, to achieve a final $\mathrm{OD}_{600}=1.0, \sim 8 \times 10^{8}$ cells $/ \mathrm{mL}$ Cells were aliquoted in $1-\mathrm{mL}$ aliquots, and stored at $-80^{\circ} \mathrm{C}$.

Flow Cytometry Analysis of Bacteria Expressing Vaccine Antigens. Approximately $5 \times 10^{7}$ cells $/ \mathrm{mL}$ were added to each well of a 96 -well V-bottom nobinding plate. Samples were blocked with phosphate-buffered saline (PBS) supplemented with $10 \%$ fetal bovine serum (FBS) for $30 \mathrm{~min}$ on ice. The plate was washed twice with PBS buffer supplemented with $2 \%$ FBS and incubated with appropriate dilution of primary antibody anti-HA (Invitrogen \#26183), custom-made anti-FP peptide antibodies (Pacific Immunology) (rabbit anti-SARS-CoV-2 FP [1:5,000 or rabbit anti-PEDV FP [1:2,000]) for $30 \mathrm{~min}$ on ice. After washing twice, samples were subsequently stained with 1:600 dilution of AlexaFluor-488 rat anti-mouse or anti-rabbit antibody (BD Biosciences) for $30 \mathrm{~min}$ on ice. Samples were examined using a FACSCalibur (BD Biosciences) flow cytometer. Data were analyzed with FlowJo $V$ software (TreeStar). Gating was set using Alexa-488 negative sample for the bacterial population by forward-scatter and side-scatter and to determine background fluorescence. The binding data are presented in Fig. $2 B$.

Immunoblots of Bacteria Expressing Vaccine Antigens. Normalized quantities of vaccines and serial dilutions of a recombinant protein, DNAK protein quantity standard (Abcam Ab51121) were resuspended in $4 \times$ Laemmli sample buffer (Bio-Rad) and incubated at $100{ }^{\circ} \mathrm{C}$ for $5 \mathrm{~min}$. Samples were separated using Novex NuPage $4-12 \%$ Bis-Tris Gel (ThermoFisher Scientific) and electrophoretically transferred onto $0.2-\mu \mathrm{m}$ nitrocellulose membranes (Bio-Rad), which were then blocked overnight in 3\% nonfat dry milk in $0.05 \%$ Tween20 in PBS (PBS-T). After washing three times, the membranes were incubated with a primary antibody (for the protein standard, mouse monoclonal antiDNAK, Abcam [8E2/2] Ab69617) at dilution of 1:2,000; or for detection of FP-AIDA-I recombinant proteins, polyclonal rabbit anti-SARS-CoV-2 FP antiserum, at a dilution of 1:4,000; or polyclonal rabbit anti-PEDV FP antiserum, at a dilution of $1: 2,000$ ) in blocking buffer for $1.5 \mathrm{~h}$ at room temperature. Membranes were then washed three times in PBS-T and incubated with
1:3,000 dilution of goat anti-mouse HRP or goat anti-rabbit-HRP (Sigma Aldrich) in blocking buffer for $1 \mathrm{~h}$ at room temperature. After washing three times again, the membranes were processed for enzyme-linked chemiluminescence using a Western Blot Signal Enhancer kit (ThermoFisher Scientific). The immunoblots signals were captured by ChemiDoc MP (Bio-Rad) and the data were analyzed in Image Lab software (Bio-Rad), and quantitated using ImageJ (https://imagej.nih.gov/ij/).

Propagation and Titration of Challenge Virus. Vero cells (African green monkey kidney cell line) cultured in DMEM (Gibco) supplemented with 10\% FBS (Gibco) were used to propagate the PEDV 2013 Colorado strain (National Veterinary Services Laboratories). After 1 -h inoculation with virus, cells were maintained in MEM (Gibco) supplemented with $0.02 \%$ yeast extraction, $0.3 \%$ tryptose phosphate broth, $2 \mu \mathrm{g} / \mathrm{mL}$ trypsin at $37{ }^{\circ} \mathrm{C}$ with $5 \% \mathrm{CO}_{2}$. Five days later, the cell lysate and culture supernatant were collected via three rounds of freeze and thaw. After centrifugation $\left(3,000 \times g, 10 \mathrm{~min}, 4{ }^{\circ} \mathrm{C}\right)$, the supernatant was collected and stored at $-80^{\circ} \mathrm{C}$ as virus stock. To determine the infectious titer $100 \mu \mathrm{L}$ serially diluted virus stock $\left(10^{-1}\right.$ to $\left.10^{-5}\right)$ was inoculated with each well of Vero cells in a 96 -well plate at $37{ }^{\circ} \mathrm{C}$ with $5 \% \mathrm{CO}_{2}$. After 3 -d incubation, the inoculated cells were analyzed via immunofluorescence assay with a PEDVspecific antibody as previously described (78). Infectious viral titer was calculated using the Reed-Muench method and expressed as $\mathrm{TCID}_{50} / \mathrm{mL}$.

Experimental Design for the Pig Vaccination and Challenge Study. A total of 21 $\mathrm{PEDV}^{-}$piglets at $5 \mathrm{wk}$ of age were randomly divided into three groups of seven piglets per group housed in separate rooms in a BSL-2 swine facility. Piglets in each group were intramuscularly injected into neck muscles with killed whole cell genome-reduced vaccines expressing SARS-CoV-2 or PEDV FPs, or killed whole bacterial cells as control (Table 1). Pigs received a booster dose at $21 \mathrm{~d}$ postvaccination (dpv). Serum samples were collected from each pig prior to vaccination and at 1, 3, 5, $6 \mathrm{wpv}$. At $35 \mathrm{dpv}$, the pigs were challenged with PEDV 2013 Colorado strain via oral route of inoculation with $3.0 \times 10^{5.0} \mathrm{TCID}_{50}$ per pig (70). After virus challenge, samples of fecal swab materials and scores of clinical signs were collected daily. At $7 \mathrm{dpc}$, all pigs were killed and necropsied. Samples of intestine tissues and intestinal contents were collected for pathological evaluation and quantification of PEDV RNA loads, respectively. This study was approved by Virginia Polytechnic Institute and State University Institutional Animal Care and Use Committee (approval no. IACUC 20-070).

Evaluation of Clinical Signs, Gross and Histological Lesions. After virus challenge, pigs were monitored daily for clinical signs of diarrhea and body condition. Diarrhea scores range from 1 to 3 , with 1 being normal to pasty feces, 2 being semiliquid diarrhea with some solid content, and 3 being liquid diarrhea with no solid content. Body condition scores range from 1 to 3 , with 1 being undetectable spinous processes and hook bones, 2 being spinous processes and hook bones were slightly felt, and 3 being spinous processes and hook bones were easily felt and visible (78).

Gross and histopathological lesions were evaluated by a board-certified veterinary pathologist (T.L.) who was blind to the treatment groups. At necropsy, small intestines were subdivided into three sections (duodenum, jejunum, and ileum), while large intestines were subdivided into two sections (cecum and colon). Gross lesions in intestine tissues were scored 1 to 3 , with 1 being normal, 2 being either thin-walled or gas-distended intestine, and 3 being both thinwalled and gas-distended intestine. Intestinal contents were also scored 1 to 3 , with 1 being solid or pasty feces, 2 being semiwatery feces, and 3 being watery feces with no solid contents. Jejunum tissues were also collected at necropsy and fixed in formalin for histological examination. H\&E-stained tissue slides were prepared from formalin-fixed tissues. Villous length $(\mathrm{V})$ and crypt depth (C) were measured at 10 different sites on each sample slide. The average $\mathrm{V}: \mathrm{C}$ was calculated. A lower V:C indicates more severe intestinal lesion.

Quantification of PEDV RNA by qRT-PCR. Total RNAs were isolated from $10 \%$ suspension of fecal swab materials, intestine contents, or samples of homogenized intestine tissues, respectively, by using TRIzol LS reagent (Thermo Fisher Scientific). The PEDV RNA loads in samples were quantitated by one step qRT-PCR kit (Bioline Sensifast Probe No Rox One Step Kit) according to the manufacturer's instruction. The primer pair, probe, and standard used in the assay were previously described $(70,79)$. The detection limit was 10 genomic copies per reaction.

Peptide-Based ELISA for Detecting Anti-PEDV FP and Anti-SARS-CoV-2 FP Antibodies. Custom-made BSA-conjugated peptides of SARS-CoV-2 FP and PEDV FP were commercially synthesized (GenScript). Ninty-six-well ELISA plates were coated with $0.2 \mu \mathrm{g} / \mathrm{mL}$ each of the BSA-conjugated peptides in 
Table 1. Experimental design for SARS-CoV-2 FP and PEDV FP vaccine efficacy study in a PEDV challenge pig model

\begin{tabular}{cccccc} 
Group & No. of pigs & Vaccination at $0 \mathrm{dpv}^{*}$ with & Booster at $21 \mathrm{dpv}^{*}$ with & Challenge at $35 \mathrm{dpv}$ with & No. of pigs at necropsy (7 dpc) $^{\dagger}$ \\
\hline 1 & 7 & ME5125 & ME5125 & PEDV & 7 \\
2 & 7 & SARS-CoV-2 FP & SARS-CoV-2 FP & PEDV & 7 \\
3 & 7 & PEDV FP & PEDV FP & PEDV & 7 \\
\hline
\end{tabular}

*Vaccination dose: SARS-CoV-2 FP $110 \mu \mathrm{g}$ per pig, PEDV FP and ME5125: $250 \mu \mathrm{g}$ per pig. Booster dose, $250 \mu \mathrm{g}$ per pig for all groups.

${ }^{\dagger}$ Challenge virus: PEDV 2013 Colorado strain, $3.0 \times 10^{5.0} \mathrm{TCID}_{50}$ per pig.

${ }^{\ddagger}$ ME5125: killed genome-reduced $E$. coli cells not expressing any vaccine antigen.

$0.05 \mathrm{M}$ carbonate-bicarbonate buffer $(\mathrm{pH} 9.6)$ at $4{ }^{\circ} \mathrm{C}$ for $12 \mathrm{~h}$. After extensive washing with Tris-buffered saline buffer with $0.05 \%$ Tween 20 (TBST), plates were blocked by blocking buffer (1.5\% BSA in TBST) at $37^{\circ} \mathrm{C}$ for $2 \mathrm{~h}$. The plates were washed, and then added with diluted serum sample (1:200 in blocking buffer). PEDV hyperimmune pig serum against $\mathrm{S} 1$ was used as positive control, while $\mathrm{PEDV}^{-}$pig serum was used as negative control (70). After incubation at $37^{\circ} \mathrm{C}$ for $1 \mathrm{~h}$, plates were washed five times and then incubated with peroxidase-conjugated rabbit anti-pig IgG $(1: 20,000$ dilution) (Milliporesigma) at $37{ }^{\circ} \mathrm{C}$ for $1 \mathrm{~h}$. After five washes, plates were developed by adding One-step Ultra TMB solution (Thermo Fisher Scientific) according to the manufacturer's instruction. The reaction was stopped by $2 \mathrm{~N}$ sulphuric acid, and the absorbance at $450 \mathrm{~nm}\left(\mathrm{OD}_{450}\right)$ was read. The normalized $\mathrm{OD}(\mathrm{S} / \mathrm{P}$ value) was calculated as $\mathrm{S} / \mathrm{P}=($ sample $\mathrm{OD}-$ negative $\mathrm{OD}) /($ positive OD - negative OD).

Generation of a Lentiviral-Based SARS-CoV-2 S Pseudovirus for Detecting Anti-SARS-CoV-2 NA. The full-length SARS-CoV-2 $S$ protein coding sequence (human codon optimized) was cloned into mammalian expression vector pcDNA under the control of a CMV-promoter with a BGH-polyA terminator. The resulting construct, pcDNA-SARS-CoV2-S, was used as a packing vector to generate pseudovirus particles containing SARS-CoV2 S. Briefly, 293T cells were transfected with firefly-luciferase-containing reporter lentivirus vector pLJM1-FFLuc, pMDLg/pRRE, pRSV-Rev (Addgene), and pcDNA-SARS-CoV2-S The transfected cells were maintained in DMEM with $10 \%$ FBS and $20 \mathrm{mM}$ Hepes at $37{ }^{\circ} \mathrm{C}$ and $5 \% \mathrm{CO}_{2}$. At $48 \mathrm{~h}$ posttransfection, cell culture supernatant containing pseudovirus-SARS-CoV2-S particles was collected and clarified using low-speed centrifugation $(2,000 \times g, 10 \mathrm{~min})$. The clarified pseudovirus preparation was then concentrated using Amicon 100-kDa filter (MilliporeSigma), and the concentrated pseudovirus (SARS-CoV2-FFLuc) was aliquoted and stored at $-80^{\circ} \mathrm{C}$ until use. The SARS-CoV2-FFLuc pseudovirus was titrated by serially diluting twofold in medium containing DMEM with $2 \%$ FBS and polybrene $(8 \mu \mathrm{g} / \mathrm{mL}) ; 100 \mu \mathrm{L}$ of the serially diluted pseudovirus was overlaid onto hACE2-overexpressing 293T (hACE2-293T) cell monolayer in a 96-well plate, and incubated at $37{ }^{\circ} \mathrm{C}$ and $5 \% \mathrm{CO}_{2}$. After $48 \mathrm{~h}$ of incubation, the luciferase expression level was estimated using Luciferase kit (Promega) per the manufacture's protocols.

To detect anti-SARS-CoV-2 NA, the heat inactivated serum samples $\left(56{ }^{\circ} \mathrm{C}\right.$ $30 \mathrm{~min}$ ) from the pig study were twofold serially diluted (starting from 1:10) and mixed with equal volume of SARS-CoV2-FFLuc lenti-pseudovirus. After 1-h incubation at $37{ }^{\circ} \mathrm{C}, 100 \mu \mathrm{L}$ of the mixtures were added to the hACE2$293 \mathrm{~T}$ cells in 96 -well plate at $90 \%$ confluence. The pseudovirus (SARS-CoV2FFLuc) only and medium only were used as controls, respectively. The plate was then incubated at $37{ }^{\circ} \mathrm{C}$ with $5 \% \mathrm{CO}_{2}$ for $48 \mathrm{~h}$. The luminescence was detected by using Luciferase kit (Promega) according to the manufacturer's instruction. After subtraction of background (medium only), samples with $\geq 50 \%$ luminescence unit reduction relative to the control (SARS-CoV2FFLuc only) was considered as positive for neutralizing antibody.

1. S. Rauch, E. Jasny, K. E. Schmidt, B. Petsch, New vaccine technologies to combat outbreak situations. Front. Immunol. 9, 1963 (2018).

2. M. Kanekiyo, D. Ellis, N. P. King, New vaccine design and delivery technologies J. Infect. Dis. 219 (suppl. 1), S88-S96 (2019).

3. B. S. Graham, J. R. Mascola, A. S. Fauci, Novel vaccine technologies: Essential components of an adequate response to emerging viral diseases. JAMA 319, 1431-1432 (2018).

4. S. Plotkin, J. M. Robinson, G. Cunningham, R. Iqbal, S. Larsen, The complexity and cost of vaccine manufacturing-An overview. Vaccine 35, 4064-4071 (2017).

5. Q. Bi et al.; Oral Cholera Vaccine Working Group of The Global Task Force on Cholera Control, Protection against cholera from killed whole-cell oral cholera vaccines: A systematic review and meta-analysis. Lancet Infect. Dis. 17, 1080-1088 (2017).

6. B. Nesta, M. Pizza, "Vaccines against Escherichia coli" in Escherichia coli, a Versatile Pathogen, G. Frankel, E. Z. Ron, Eds. (Springer International Publishing, Cham, 2018), pp. 213-242.
High-Throughput Neutralization Test for Detecting Anti-PEDV NA. To detect the NA against PEDV, pig sera were tested using a high-throughput neutralization test assay at the lowa State University Veterinary Diagnostic Laboratory (80). Briefly, 1:20 diluted heat-inactivated serum samples were mixed with a fixed amount of PEDV at 1:1 volume ratio (final serum dilution 1:40). The serum-virus mixtures were inoculated onto Vero cells in 96-well plate for 1.5 to $2 \mathrm{~h}$ at $37^{\circ} \mathrm{C}$. After adding fresh culture medium, cells were incubated for $24 \mathrm{~h}$, then fixed and stained with a conjugated PEDV monoclonal antibody, followed by reading on image cytometry. The 1:40 diluted serum samples with a $\geq 85 \%$ total fluorescence reduction ( $\% \mathrm{FR}$ ) relative to the control were classified as positive for NA.

Detection of IFN- $\gamma$ in Pig Sera. The level of IFN- $\gamma$ in pig serum samples was evaluated by using a commercial Swine IFN- $\gamma$ ELISA Kit (MyBioSource) according to the manufacturer's instruction. Briefly, twofold serially diluted IFN- $\gamma$ standard ( $500 \mathrm{pg} / \mathrm{mL}$ to $7.8 \mathrm{pg} / \mathrm{mL}$ ) and undiluted pig serum samples were added into a 96-well microplate precoated with IFN- $\gamma$-specific antibody. After 1 -h incubation at $37{ }^{\circ} \mathrm{C}$, the plate was aspirated and added with a Biotin-conjugated antibody. The plate was incubated at $37{ }^{\circ} \mathrm{C}$ for $1 \mathrm{~h}$ and subsequently washed three times. Streptavidin-HRP was added into plate and incubated for $30 \mathrm{~min}$ at $37{ }^{\circ} \mathrm{C}$. After five washes, the plate was developed by adding TMB substrate at $37{ }^{\circ} \mathrm{C}$ for $15 \mathrm{~min}$ prior to the addition of stop solution. The $\mathrm{OD}_{450}$ was read using a microplate reader. All the reagents used in this assay are included in this kit.

Statistical Analysis. Statistical analysis was done using R (v1.3.1093) with the Rstudio environment with included packages and the tidyverse and stats packages, with visualizations using ggplot2.

Data Availability. The sequence of pRAIDA2 has been deposited into GenBank (accession no. MW383928) https://www.ncbi.nlm.nih.gov/nuccore/MW383928. All other study data are included in the article and supporting information.

ACKNOWLEDGMENTS. We thank Amy Rizzo, Karen Hall, Cassie Fields, and Jeff Estienne for their support of the animal study; and Barbie GanserPornillos and Owen Pornillos for structural biology advice. The work was supported through the Pendleton Pediatric Infectious Disease Laboratory, by the funding provided to S.L.Z. via the McClemore Birdsong endowed chair, and by support from the University of Virginia Manning Fund for COVID-19 Research and from the Ivy Foundation. The work is also supported by the Virginia-Maryland College of Veterinary Medicine (FRS\#175420), and Virginia Polytechnic Institute and State University internal funds FRS\#440783. We thank Claude Chew and the University of Virginia Flow Cytometry Core for excellent flow cytometry assistance.

7. A. Gohar, N. F. Abdeltawab, A. Fahmy, M. A. Amin, Development of safe, effective and immunogenic vaccine candidate for diarrheagenic Escherichia coli main pathotypes in a mouse model. BMC Res. Notes 9, 80 (2016).

8. J. Holmgren et al., Development and preclinical evaluation of safety and immunogenicity of an oral ETEC vaccine containing inactivated $\mathrm{E}$. coli bacteria overexpressing colonization factors CFA/l, CS3, CS5 and CS6 combined with a hybrid LT/CT B subunit antigen, administered alone and together with dmLT adjuvant. Vaccine 31, 2457-2464 (2013).

9. M. P. Hays, A. C. Ericsson, Y. Yang, P. R. Hardwidge, Vaccinating with conserved Escherichia coli antigens does not alter the mouse intestinal microbiome. BMC Res. Notes 9, 401 (2016).

10. D. G. Evans, D. J. Evans Jr, A. R. Opekun, D. Y. Graham, Non-replicating oral whole cell vaccine protective against enterotoxigenic Escherichia coli (ETEC) diarrhea: Stimulation of anti-CFA (CFA/I) and anti-enterotoxin (anti-LT) intestinal IgA and protection against challenge with ETEC belonging to heterologous serotypes. FEMS Microbiol. Immunol. 1, 117-125 (1988). 
11. S. J. Savarino et al.; PRIDE Study Group, Oral, inactivated, whole cell enterotoxigenic Escherichia coli plus cholera toxin B subunit vaccine: Results of the initial evaluation in children. J. Infect. Dis. 179, 107-114 (1999).

12. S. A. Selim et al., The effect of Escherichia coli $J 5$ and modified live Salmonella dublin vaccines in artificially reared neonatal calves. Vaccine 13, 381-390 (1995).

13. H. A. Deluyker, P. Rossitto, S. N. Van Oye, J. S. Cullor, Efficacy of an Escherichia coli J-5 mutant strain bacterin in the protection of calves from endotoxin disease caused by subcutaneous challenge with endotoxins from Escherichia coli. Vaccine 23, 709-717 (2004).

14. L. Odevall et al., The Euvichol story-Development and licensure of a safe, effective and affordable oral cholera vaccine through global public private partnerships. Vaccine 36, 6606-6614 (2018)

15. E. van Bloois, R. T. Winter, H. Kolmar, M. W. Fraaije, Decorating microbes: Surface display of proteins on Escherichia coli. Trends Biotechnol. 29, 79-86 (2011).

16. T. Nicolay, J. Vanderleyden, S. Spaepen, Autotransporter-based cell surface display in gram-negative bacteria. Crit. Rev. Microbiol. 41, 109-123 (2013).

17. I. R. Henderson, R. Cappello, J. P. Nataro, Autotransporter proteins, evolution and redefining protein secretion. Trends Microbiol. 8, 529-532 (2000).

18. J. Jose, T. F. Meyer, The autodisplay story, from discovery to biotechnical and biomedical applications. Microbiol. Mol. Biol. Rev. 71, 600-619 (2007)

19. W. S. Jong et al., A structurally informed autotransporter platform for efficient heterologous protein secretion and display. Microb. Cell Fact. 11, 85 (2012).

20. H. Chen, D. M. Schifferli, Comparison of a fimbrial versus an autotransporter display system for viral epitopes on an attenuated Salmonella vaccine vector. Vaccine 25, 1626-1633 (2007).

21. K. Kjaergaard, H. Hasman, M. A. Schembri, P. Klemm, Antigen 43-mediated autotransporter display, a versatile bacterial cell surface presentation system. J. Bacteriol. 184, 4197-4204 (2002).

22. K. Rizos, C. T. Lattemann, D. Bumann, T. F. Meyer, T. Aebischer, Autodisplay. Efficacious surface exposure of antigenic UreA fragments from Helicobacter pylori in Salmonella vaccine strains. Infect. Immun. 71, 6320-6328 (2003).

23. F. Ruiz-Pérez et al., Expression of the Plasmodium falciparum immunodominant epitope (NANP)(4) on the surface of Salmonella enterica using the autotransporter MisL. Infect. Immun. 70, 3611-3620 (2002).

24. J. Kato, M. Hashimoto, Construction of consecutive deletions of the Escherichia coli chromosome. Mol. Syst. Biol. 3, 132 (2007).

25. M. Hashimoto et al., Cell size and nucleoid organization of engineered Escherichia coli cells with a reduced genome. Mol. Microbiol. 55, 137-149 (2005).

26. F. Amanat, F. Krammer, SARS-CoV-2 vaccines: Status report. Immunity 52, 583-589 (2020).

27. L. Corey, J. R. Mascola, A. S. Fauci, F. S. Collins, A strategic approach to COVID-19 vaccine R\&D. Science 368, 948-950 (2020)

28. L. M. Yonker et al., Pediatric SARS-CoV-2: Clinical presentation, infectivity, and immune responses. J. Pediatr. 227, 45-52.e5. (2020)

29. C. A. Rostad et al., Quantitative SARS-CoV-2 serology in children with multisystem inflammatory syndrome (MIS-C). Pediatrics 146, e2020018242. (2020)

30. S. L. Zeichner, A. T. Cruz, Multisystem inflammatory syndrome in children and SARS-CoV-2 serology. Pediatrics 146, e2020032888. (2020).

31. P. M. Colman, M. C. Lawrence, The structural biology of type I viral membrane fusion Nat. Rev. Mol. Cell Biol. 4, 309-319 (2003).

32. R. Kong et al., Fusion peptide of HIV-1 as a site of vulnerability to neutralizing an tibody. Science 352, 828-833 (2016)

33. K. Xu et al., Epitope-based vaccine design yields fusion peptide-directed antibodies that neutralize diverse strains of HIV-1. Nat. Med. 24, 857-867 (2018).

34. M. J. van Gils et al., An HIV-1 antibody from an elite neutralizer implicates the fusion peptide as a site of vulnerability. Nat. Microbiol. 2, 16199 (2016).

35. A. L. Lai, J. K. Millet, S. Daniel, J. H. Freed, G. R. Whittaker, The SARS-CoV fusion peptide forms an extended bipartite fusion platform that perturbs membrane order in a calcium-dependent manner. J. Mol. Biol. 429, 3875-3892 (2017).

36. T. Tang, M. Bidon, J. A. Jaimes, G. R. Whittaker, S. Daniel, Coronavirus membrane fusion mechanism offers a potential target for antiviral development. Antiviral Res. 178, 104792 (2020).

37. E. A. J. Alsaadi, B. W. Neuman, I. M. Jones, A fusion peptide in the spike protein of MERS coronavirus. Viruses 11, 825 (2019).

38. C. M. Poh et al., Two linear epitopes on the SARS-CoV-2 spike protein that elicit neutralising antibodies in COVID-19 patients. Nat. Commun. 11, 2806 (2020).

39. A. C. Walls et al., Structure, function, and antigenicity of the SARS-CoV-2 spike gly coprotein. Cell 181, 281-292.e6 (2020).

40. J. A. Jaimes, N. M. André, J. S. Chappie, J. K. Millet, G. R. Whittaker, Phylogenetic analysis and structural modeling of SARS-CoV-2 spike protein reveals an evolutionary distinct and proteolytically sensitive activation loop. J. Mol. Biol. 432, 3309-3325 (2020).

41. L. van Dorp et al., Emergence of genomic diversity and recurrent mutations in SARS-CoV-2. Infect. Genet. Evol. 83, 104351 (2020).

42. K. W. Ng et al., Preexisting and de novo humoral immunity to SARS-CoV-2 in humans. Science 370, 1339-1343 (2020).

43. E. Shrock et al.; MGH COVID-19 Collection \& Processing Team, Viral epitope profiling of COVID-19 patients reveals cross-reactivity and correlates of severity. Science 370 eabd4250 (2020).

44. T. Miyoshi-Akiyama et al., Fully human monoclonal antibody directed to proteolytic cleavage site in severe acute respiratory syndrome (SARS) coronavirus $S$ protein neutralizes the virus in a rhesus macaque SARS model. J. Infect. Dis. 203, 1574-1581 (2011).

45. S. H. Sun et al., A mouse model of SARS-CoV-2 infection and pathogenesis. Cell Host Microbe 28, 124-133.e4 (2020).
46. P. B. McCray Jr et al., Lethal infection of K18-hACE2 mice infected with severe acute respiratory syndrome coronavirus. J. Virol. 81, 813-821 (2007)

47. S. F. Sia et al., Pathogenesis and transmission of SARS-CoV-2 in golden hamsters. Nature 583, 834-838 (2020)

48. L. M. Gretebeck, K. Subbarao, Animal models for SARS and MERS coronaviruses. Curr. Opin. Virol. 13, 123-129 (2015).

49. M. D. Johansen et al., Animal and translational models of SARS-CoV-2 infection and COVID-19. Mucosal Immunol. 13, 877-891 (2020)

50. Y. Zhang et al., Coagulopathy and antiphospholipid antibodies in patients with covid19. N. Engl. J. Med. 382, e38 (2020).

51. R. Hanafi et al., COVID-19 neurologic complication with CNS vasculitis-like pattern AJNR Am. J. Neuroradiol. 41, 1384-1387 (2020).

52. L. Jiang et al., COVID-19 and multisystem inflammatory syndrome in children and adolescents. Lancet Infect. Dis. 20, e276-e288 (2020).

53. G. W. Stevenson et al., Emergence of porcine epidemic diarrhea virus in the United States: Clinical signs, lesions, and viral genomic sequences. J. Vet. Diagn. Invest. $\mathbf{2 5}$ 649-654 (2013)

54. Y.-W. Huang et al., Origin, evolution, and genotyping of emergent porcine epidemic diarrhea virus strains in the United States. MBio 4, e00737-13 (2013).

55. K. Jung, L. J. Saif, Porcine epidemic diarrhea virus infection: Etiology, epidemiology, pathogenesis and immunoprophylaxis. Vet. J. 204, 134-143 (2015)

56. F. Meurens, A. Summerfield, H. Nauwynck, L. Saif, V. Gerdts, The pig: A model for human infectious diseases. Trends Microbiol. 20, 50-57 (2012).

57. J. Gu, B. Han, J. Wang, COVID-19: Gastrointestinal manifestations and potential fecaloral transmission. Gastroenterology 158, 1518-1519 (2020)

58. L. Jiao et al., The gastrointestinal tract is an alternative route for SARS-CoV-2 infection in a nonhuman primate model. Gastroenterology, 10.1053/j.gastro.2020.12.001 (2020).

59. J. Cohen, Vaccine wagers on coronavirus surface protein pay off. Science 370, 894-895 (2020).

60. J.-S. Lee et al., Evidence of severe acute respiratory syndrome coronavirus 2 reinfection after recovery from mild coronavirus disease 2019. Clin. Infect. Dis., 10.1093/cid/ ciaa1421 (2020).

61. J. Van Elslande et al., Symptomatic SARS-CoV-2 reinfection by a phylogenetically distinct strain. Clin. Infect. Dis., 10.1093/cid/ciaa1330 (2020)

62. E. Volz et al., Evaluating the effects of SARS-CoV-2 spike mutation D614G on transmissibility and pathogenicity. Cell 184, 64-75.e11 (2020).

63. S. Kemp et al., Recurrent emergence and transmission of a SARS-CoV-2 Spike deletion $\Delta$ H69/AV70. bioRxiv [Preprint] (2020). https://www.biorxiv.org/content/10.1101/2020. 12.14.422555v2 (Accessed 5 April 2021).

64. A. J. Greaney et al., Complete mapping of mutations to the SARS-CoV-2 spike receptor-binding domain that escape antibody recognition. Cell Host Microbe 29, 44-57.e9 (2020).

65. T. N. Starr et al., Prospective mapping of viral mutations that escape antibodies used to treat COVID-19. bioRxiv [Preprint] (2020). https://www. biorxiv.org/content/10.1101/ 2020.11.30.405472v1 (Accessed 5 April 2021).

66. S. A. Kemp et al., Neutralising antibodies drive Spike mediated SARS-CoV-2 evasion. medRxiv [Preprint] (2020). https://www.medrxiv.org/content/10.1101/2020.12.05. 20241927v1 (Accessed 5 April 2021).

67. Q. Li et al., The impact of mutations in SARS-CoV-2 spike on viral infectivity and antigenicity. Cell 182, 1284-1294.e9 (2020).

68. E. C. Thomson et al.; ISARIC4C Investigators; COVID-19 Genomics UK (COG-UK) Consortium, Circulating SARS-CoV-2 spike N439K variants maintain fitness while evading antibody-mediated immunity. Cell 184, 1171-1187.e20 (2021).

69. Z. Wang et al., mRNA vaccine-elicited antibodies to SARS-CoV-2 and circulating variants. bioRxiv [Preprint] (2021). https://www.biorxiv.org/content/10.1101/2021.01.15. 426911v1 (Accessed 5 April 2021)

70. S. Subramaniam et al., Vaccination of sows with a dendritic cell-targeted porcine epidemic diarrhea virus $\mathrm{S} 1$ protein-based candidate vaccine reduced viral shedding but exacerbated gross pathological lesions in suckling neonatal piglets. J. Gen. Virol. 99, 230-239 (2018).

71. M. Kang et al., Probable evidence of fecal aerosol transmission of SARS-CoV-2 in a high-rise building. Ann. Intern. Med. 173, 974-980 (2020).

72. D. A. Larsen, K. R. Wigginton, Tracking COVID-19 with wastewater. Nat. Biotechnol. 38, 1151-1153 (2020)

73. C. A. Guzman et al., Vaccines against typhoid fever. Vaccine 24, 3804-3811 (2006).

74. I. R. Tizard, Vaccination against coronaviruses in domestic animals. Vaccine $\mathbf{3 8}$, 5123-5130 (2020)

75. A. Pratelli et al., Safety and efficacy of a modified-live canine coronavirus vaccine in dogs. Vet. Microbiol. 99, 43-49 (2004).

76. K. McMahan et al., Correlates of protection against SARS-CoV-2 in rhesus macaques. Nature 590, 630-634 (2020)

77. Duke Global Health Innovation Center, Launch and scale speedometer (Duke Global Health Innovation Center, Durham, NC, 2020), https://launchandscalefaster.org/ COVID-19. Accessed 5 April 2021.

78. Y. Lu et al., Virus-like particle vaccine with B-cell epitope from porcine epidemic diarrhea virus (PEDV) incorporated into hepatitis B virus core capsid provides clinical alleviation against PEDV in neonatal piglets through lactogenic immunity. Vaccine 38 5212-5218 (2020)

79. T. Opriessnig, C. T. Xiao, P. F. Gerber, J. Zhang, P. G. Halbur, Porcine epidemic diarrhea virus RNA present in commercial spray-dried porcine plasma is not infectious to naïve pigs. PLoS One 9, e104766 (2014).

80. L. V. Sarmento et al., Detection of porcine epidemic diarrhea virus-neutralizing antibody using high-throughput imaging cytometry. J. Vet. Diagn. Invest. 32 324-328 (2020) 